\title{
Analysis of the Ub to Ub-CR transition in ubiquitin
}

\author{
Konstantin Röder* and David J. Wales* \\ Department of Chemistry, University of Cambridge, Lensfield Road, Cambridge, CB2 \\ $1 E W, U K$ \\ E-mail: kr366@cam.ac.uk; dw34@cam.ac.uk
}

\begin{abstract}
A new conformation has recently been reported for ubiquitin (Ub). This invisible conformation (Ub-CR), where the C-terminal tail is retracted, has a key functional role in phosphorylation of the Ser65 residue, a trigger for PINK1 and Parkin dependent mitophagy. Here we calculate the transition mechanism and associated rates for the Ub to Ub-CR pathway in the wild type protein and a selection of mutants. We predict a cooperative one-step process with a transition state that resembles the Ub configuration, characterised by loss of all interactions of the C-terminal tail with surrounding residues, and an open ubiquitin scaffold. The calculated observables agree well with reported values, and we make a range of predictions to guide future experiments. In particular, the effect of mutations on the pathway and the corresponding structural ensembles should have observable consequences. This crosstalk between theory and experiment promises new insight into key cellular processes.
\end{abstract}

\footnotetext{
${ }^{*}$ To whom correspondence should be addressed
} 


\section{Introduction}

The ubiquitination of proteins in cells provides a highly tunable biochemical signal, with a variety of distinct functions. ${ }^{1}$ This variability manifests itself in the existence of polyubiquitin chains with alternative structures and functionality, ${ }^{1}$ as well as in the post-translational modifications of ubiquitin itself. ${ }^{2-4}$ However, the actual interactions of these modified forms of ubiquitin within biological systems are poorly understood. An exception is the phosphorylation of ubiquitin at Ser65, which is linked to mitophagy, the selective degradation of mitochondria. ${ }^{5-8}$

Phosphorylation at Ser65 is achieved by the kinase PINK1, ${ }^{9-12}$ but until recently this process was poorly understood, as the canonical ubiquitin fold (Ub) does not expose Ser65, and furthermore, the residue forms hydrogen bonds with Gln62. ${ }^{13}$ Recently it was shown that ubiquitin exhibits another conformation, ${ }^{13}$ which had previously only been observed for phosphorylated ubiquitin. ${ }^{12,14}$ In the newly described conformation (Ub-CR) the C-terminal tail is retracted, exposing Ser65. Although sparsely populated, the Ub-CR conformation is the target of phosphorylation by PINK1, ${ }^{15}$ and perturbation of this event leads to hereditary forms of Parkinson's disease. ${ }^{16}$ Interestingly, the resulting phosphorylated ubiquitin also exhibits a higher propensity for the Ub-CR conformation. ${ }^{12,14}$ While the existence of the Ub-CR conformation provides insight into the phosphorylation at Ser65, it is not known how the transition between Ub and Ub-CR occurs.

Here we show how analysis of the underlying energy landscape provides access to the Ub to Ub-CR transition mechanism in atomistic detail. The study by Gladkova et al. describes a number of mutations and their effect on the equilibrium between Ub and Ub-CR. The corresponding energy landscapes should reflect the changes caused by these mutations, providing further insight into the experimental results, and verification of our predicted mechanism. As the two states of Ub fulfil different biological functions, and are significantly different in structure, we expect them to correspond to different funnels on the energy landscape. Consequently, enhanced sampling techniques are necessary to characterise the interconversion 
pathway. Here, we apply the computational potential energy landscape framework, which is based on geometry optimisation and post-processing using tools of statistical mechanics and unimolecular rate theory, to the wild type ubiquitin and four mutants, namely L67S, L71Y, F4A and T66V + L67N, all of which have been studied experimentally. We identify an effective one step mechanism with Arrhenius temperature dependence, where the overall free energy barrier corresponds to the loss of all interactions of the C-terminal tail with its surroundings, which implies opening of the ubiquitin scaffold. Our results are in good agreement with the experimental exchange rates and occupation probabilities, and provide detailed explanations and predictions for the pathways and the effect of mutations.

\section{Methods}

Our calculations are based on the computational potential energy landscape framework, which is founded on geometry optimisation with post-processing using the tools of statistical mechanics and unimolecular rate theory to predict structure, dynamics and thermodynamics.

Discrete path sampling ${ }^{17,18}$ was employed to construct kinetic transition networks. ${ }^{19,20}$ The doubly-nudged elastic band algorithm, ${ }^{21-23}$ coupled with hybrid eigenvector-following, ${ }^{24}$ was used to locate transition states. The minima connected by the transition states were then characterised using approximate steepest-decent pathways. Free energies were calculated using the superposition approach within a harmonic approximation, ${ }^{25}$ and the definition of free energy states employed a recursive regrouping scheme. ${ }^{26}$ Graphical representations of the energy landscapes use disconnectivity graphs; ${ }^{27,28}$ with further details available in several reviews. ${ }^{20,29}$

\section{Force field and starting points}

The symmetrised AMBER ff14SB ${ }^{30}$ force field with an implicit, generalised Born solvation $^{31,32}$ model was used, in combination with the Debye-Hückel approximation ${ }^{33}$ for ions 
(0.1M). The calculations were run on GPUs using the appropriate AMBER12 ${ }^{34,35}$ and OPTIM implementations. ${ }^{36}$ The starting points for path sampling were the crystal structures for the wild type Ub conformation (PDB entry 1UBQ) ${ }^{37}$ and the L67S mutant Ub-CR conformation (PDB entry 5OXI). ${ }^{13}$ The structures were mutated using LEAP to yield one Ub and one Ub-CR structure for all five sequences.

\section{Free energy barrier estimates}

Using the occupation probability, $p_{\mathrm{UbCR}}$, and the exchange rate reported from experiment, the rate constant may be calculated as ${ }^{38}$

$$
k_{\mathrm{Ub} \leftarrow \mathrm{UbCR}}=k_{\mathrm{ex}} p_{\mathrm{UbCR}} .
$$

Assuming a one-step process, Arrhenius behaviour is expected, allowing us to estimate the experimental free energy barrier as

$$
\Delta G_{\exp }^{\ddagger}=-R T \ln \left(\frac{h}{k_{B} T} k_{\mathrm{Ub} \leftarrow \mathrm{UbCR}}\right)=-R T \ln \left(\frac{h}{k_{B} T} k_{\mathrm{ex}} p_{\mathrm{UbCR}}\right) .
$$

Before the rate constants and the free energy barriers can be computed, the landscape has to be partitioned correctly into reactant and product sets. We employed a simple order parameter, $q$, which is the ratio of the distances between the $\alpha$-C atoms of residues 4 and 65 ,

and 4 and 67 , to diagnose the required partition. The experimental data ${ }^{13}$ corresponds to NMR experiments, and the key difference between conformations is the retracted C-terminus. We therefore group all Ub-CR conformations together, including the alternative structures described, as a single experimental state. 


\section{Results and discussion}

First we focus on the wild type sequence and describe the free and potential energy landscapes, the structural ensembles they correspond to, and the transition mechanism connecting $\mathrm{Ub}$ and $\mathrm{Ub}-\mathrm{CR}$.

\section{Energy landscape for the wild type}

The potential energy landscape for the wild type is shown in Fig. 1. The overall structure consists of two funnels, one for $\mathrm{Ub}$ and one for $\mathrm{Ub}-\mathrm{CR}$, as expected from the two-state behaviour observed in experiment. ${ }^{13}$ There are no intermediate funnels, indicating the absence of stable intermediate structures, and a concerted interconversion process, rather than a step-wise retraction. This interpretation is supported by an analysis of the solvent exposure of the backbone and side chains for residues 64 to 72 (see supplementary information).

The Ub-CR funnel exhibits some substructure, but the corresponding energy barriers are significantly smaller than the overall energy barrier between Ub and Ub-CR, leading to a separation in time scales between the rate determining step from Ub to Ub-CR, and the interconversion within the alternative Ub-CR conformations. The Ub and Ub-CR structures we predict closely match the reported configurations from crystallographic experiments (see supplementary information). We find a range of populated Ub-CR structures, and the most favourable of these matches experiment. Two alternative motifs exhibit perturbations to the folding pattern in the Ser65 region. The first set of these motifs exhibit interaction of the Ser65 side chain with the preceding helical region, leading to a enthalpically favoured, but entropically disfavoured retracted conformation, where Ser65 is not exposed. The second set has misalignments in the hydrophobic pockets, with Leu67 not moving into the pocket vacated by Ser65. This change shifts Ser65 towards Phe4, preventing the formation of a longer helical region, and leads to reduced exposure of Ser65. Due to the hindered formation of a helix and the additional interactions of Ser65 with Phe4, there are entropic and 


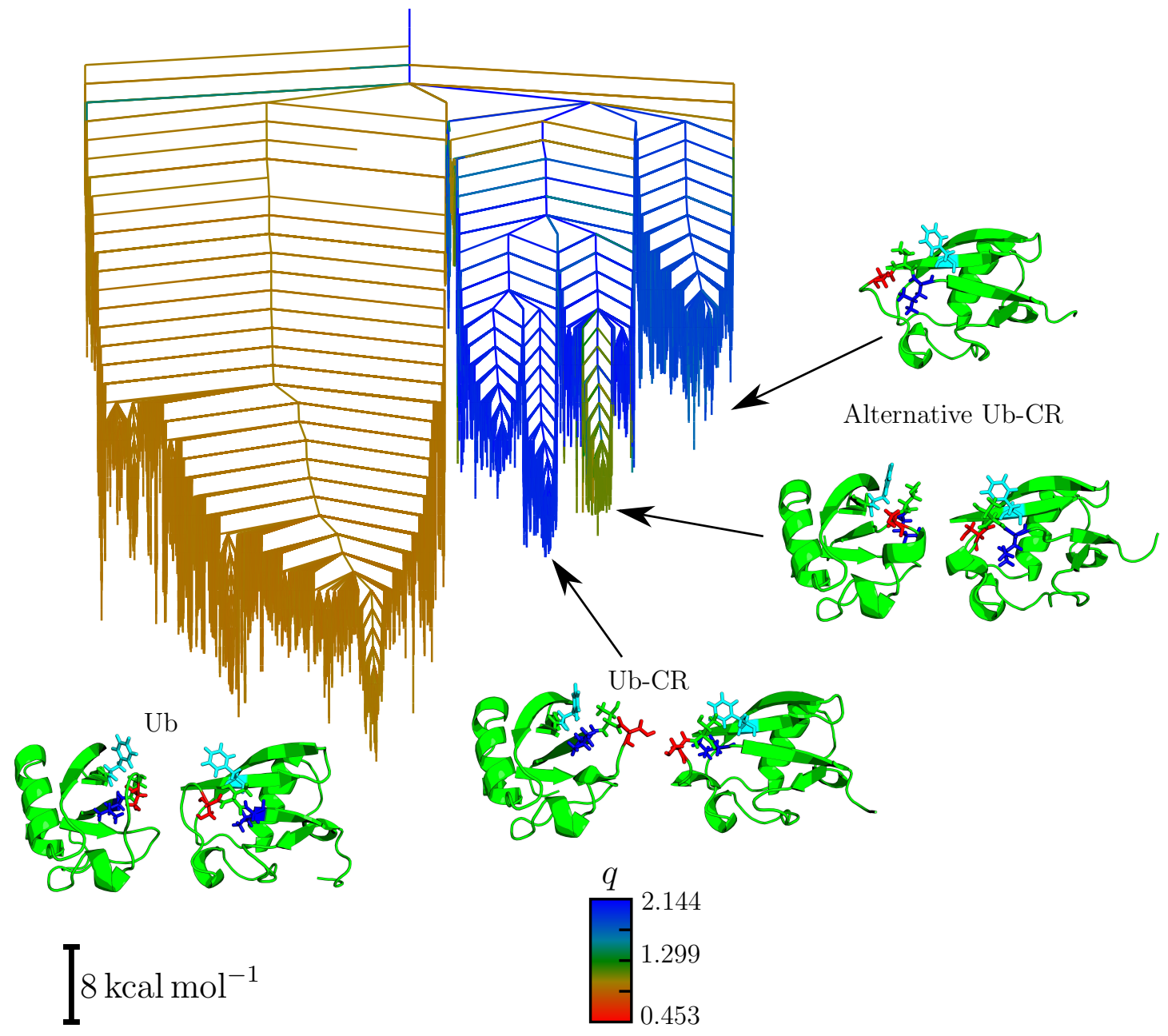

Figure 1: The disconnectivity graph for the potential energy landscape of the native sequence for ubiquitin. The canonical conformation (Ub) and the retracted C-terminal configurations (Ub-CR) are distinguished by the order parameter $q$. 
enthalpic factors stabilising this structure, leading to an interesting transition in the free energy landscapes and the pathways between Ub and Ub-CR.

In Fig. 2 the free energy landscape for the native sequence is shown at $35^{\circ} \mathrm{C}$ and $45^{\circ} \mathrm{C}$ alongside the changes in free energies for the overall one-step transition. Below $36{ }^{\circ} \mathrm{C}$, we observe a preference for the second set of alternative structures, which changes to a preference for the canonical Ub-CR configurations at higher temperatures. This behaviour results in a significant drop in the free energy and hence stabilisation of Ub-CR. Interestingly, Gladkova et al. were only able to detect $\mathrm{Ub}-\mathrm{CR}$ for the wild type in the higher temperature regime. While the transition temperature should result in a higher equilibrium occupation probability in vivo, experiments at lower temperatures may not be able to detect these configurations; in particular, phosphorylation should be slower.

\section{Mechanism for the Ub to Ub-CR transformation}

As shown in Fig. 2, the mechanism may be represented as a single main step, as illustrated in Fig. 3. The transition state exhibits a Ub-like conformation for the C-terminal tail. However, the scaffold has opened up, with changes in the $\beta 1 / \beta 2$-loop, in residues 72 to 76 , and in the interactions of the $\beta$-strand residues above $(4,6$ and 8 ) and below (42 and 44) the $\beta 5$ strand. These changes result in the loss of all interactions, so there is no energetic barrier to retraction, and the relaxation of the scaffold afterwards is energetically favourable. Analysing the pathway in more detail reveals the participation of individual residues (see supporting information).

In the Ub configuration there are significant interactions of the side chain of residue 72 with residues 51 to 53 , and the $\beta 1 / \beta 2$-loop is close to the $\alpha 1$-helix. Displacement of the $\beta 1 / \beta 2$-loop away from the $\alpha 1$-helix opens space, allowing for more flexibility of the C-

terminus. This motion is likely, as the $\beta 1 / \beta 2$-loop has been found to be the most flexible region in ubiquitin. ${ }^{39}$ Additional displacements of residues 71 to 76 bring the side chain of Leu71 into this region, and into the vicinity of Leu69. As the motion of the $\beta 1 / \beta 2$-loop 


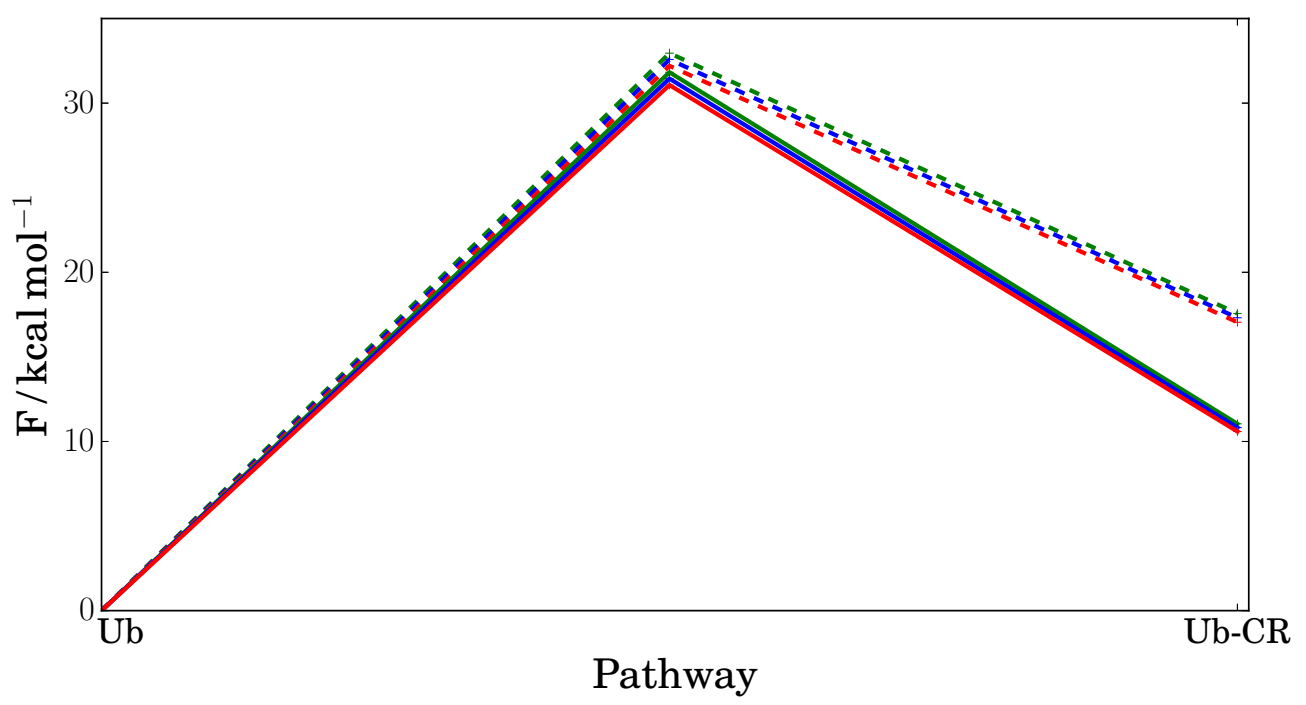

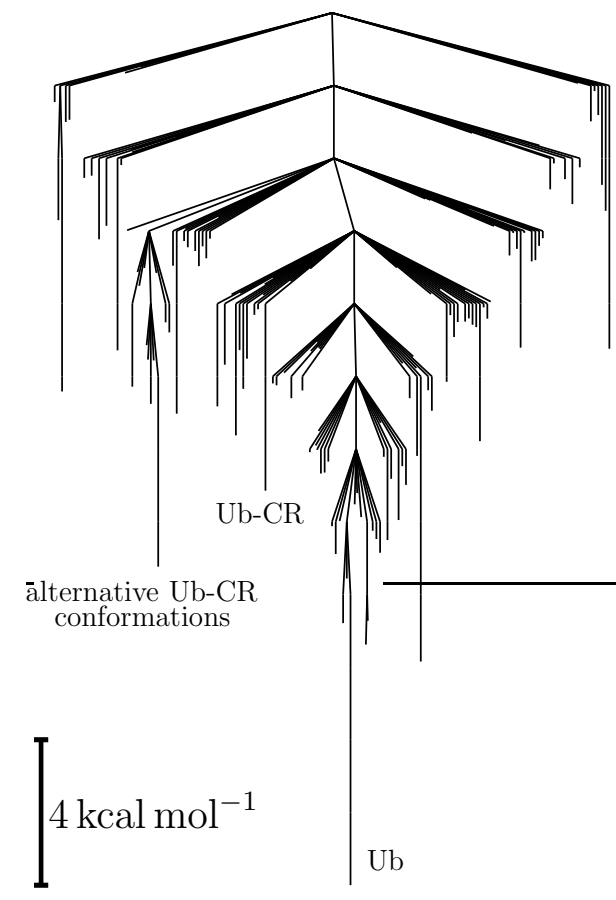

$35{ }^{\circ} \mathrm{C}$

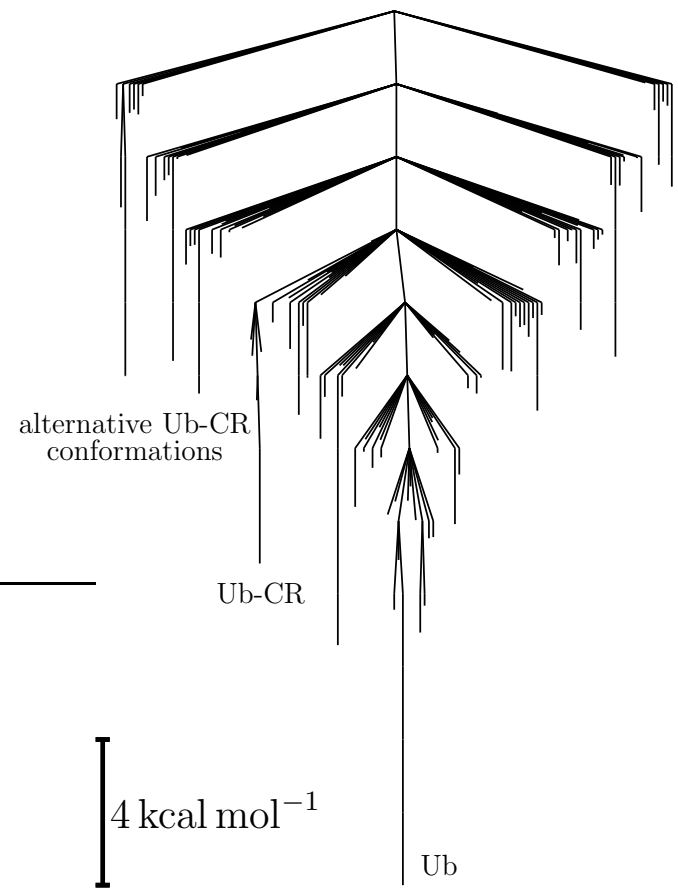

$45^{\circ} \mathrm{C}$

Figure 2: Free energy reaction profiles (top) and the free energy landscape at at $35^{\circ} \mathrm{C}$ and $45^{\circ} \mathrm{C}$ for the native sequence. For temperatures below $36^{\circ} \mathrm{C}$ (dashed lines), the most favourable Ub-CR configuration is the second set of alternative configurations described, leading to a a lowered occupation probability. Above this temperature (solid lines) the canonical structure is favoured, resulting in a drop in free energy and a higher occupation probability. The different colours correspond to $25^{\circ} \mathrm{C}, 30^{\circ} \mathrm{C}$ and $35^{\circ} \mathrm{C}$, and $40^{\circ} \mathrm{C}, 45^{\circ} \mathrm{C}$ and $50^{\circ} \mathrm{C}$ for the low and high temperature cases, respectively, with little variation observed. The free energy disconnectivity graphs highlight the different relative energies of the Ub-CR structures at these temperatures. 

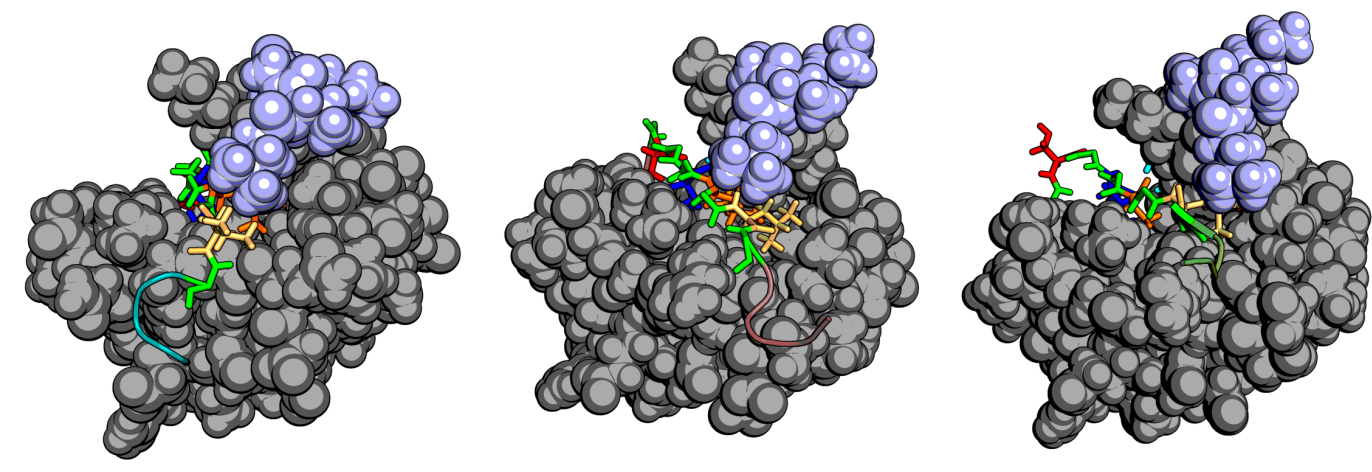

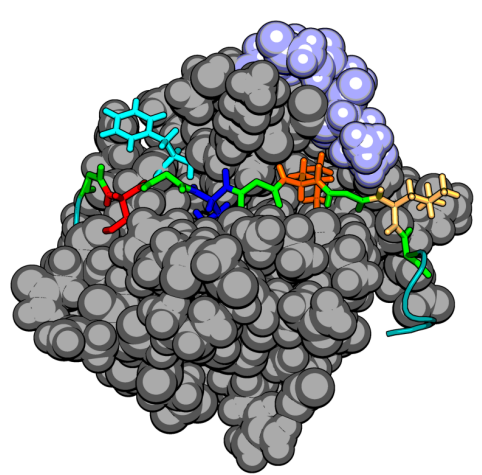

Ub configuration

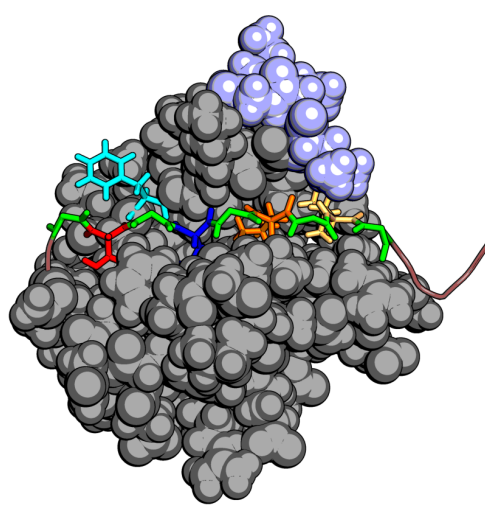

Transition state

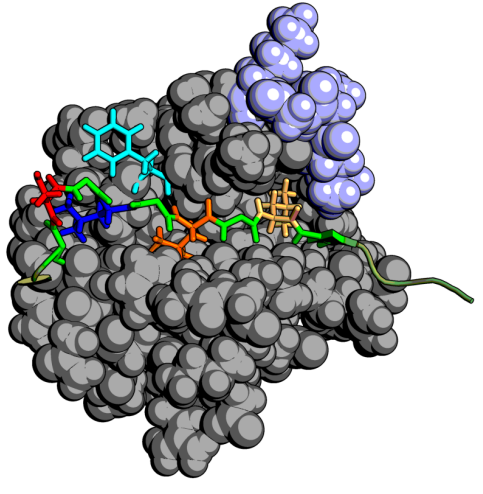

Ub-CR configuration

Figure 3: Structures of two free energy minima and the transition state for the Ub to Ub-CR pathway. The C-terminal tail is shown in a stick representation with Ser65 in red and Leu67 in blue. The $\beta 1 / \beta 2$-loop is coloured in purple. The transition state is similar to Ub, but differs in the $\beta 1 / \beta 2$-loop and the opening of the scaffold, as seen in the structure. 
enlarges the hydrophobic pockets, Leu69 can move closer to Leu67 by a side chain rotation, forcing a backbone rotation that breaks the interactions between residues 42 and 44, and 70 and 72. As a result, residues 68 to 76 lose all interactions with other residues outside the Cterminal tail at this stage; however, Leu67 and Ser65 are still in their original environments.

In the next step, backbone motion breaks the Ser65-Gln62 interaction and pulls Leu67 away from Phe4. Although the residues are still in their approximate Ub positions, the entire strand has been pulled out, and the scaffold around it has opened like a clam. At this point fluctuations can lead to two outcomes: either Leu67 moves back towards Phe4, pushing the strand back towards Ub, or it moves further out of its pocket, pulling Leu69 and Leu71 along. Pulling brings residues 70 and 72 close to residues 42 and 44, resulting in the reformation of a $\beta$-sheet, stabilising the new conformation. Residues 68 to 76 are now in their expected positions, but the region around Ser65 and Leu67 is disordered, allowing for the formation of the alternative Ub-CR structures described above. Further displacement of the backbone in this region will eventually lead to the formation of native contacts and the stable structural motif, exposing Ser65.

From these predictions, a number of key features in the Ub to Ub-CR transition can be highlighted. The mechanism requires dissociation of the strands starting around residues 69 and 71 , with displacement of the $\beta 1 / \beta 2$-loop. Introducing stronger interaction, for example the L71Y mutation, should increase the energy barrier for this process significantly, as it prevents the opening of the scaffold, which is a key element of the concerted process. Finally, the concerted mechanism requires all interactions between the C-terminal strand and its surroundings to be lost.

\section{Energy barriers}

From the occupation probabilities and the exchange rates reported, it is possible to estimate the free energy barrier of the Ub-CR to Ub transition. From the data of Gladkova et al., assuming Arrhenius behaviour (see supporting information), this estimate is $19.32 \mathrm{kcal} \mathrm{mol}^{-1}$ 
at $45^{\circ} \mathrm{C}$. If we group all Ub-CR configurations together as the experimental Ub-CR state, including the alternative structures, we calculate a free energy from the energy landscape of $20.63 \mathrm{~mol}^{-1}$ at this temperature. Considering the use of an empirical force field and implicit solvent, this agreement is well within the expected systematic error, showing that our proposed mechanism is consistent with experiment.

\section{Energy landscapes for the mutants}

The mutants studied here were employed by Gladkova et. al ${ }^{13}$ to probe the equilibrium between Ub and Ub-CR. The potential energy disconnectivity graphs ${ }^{27,28}$ for the mutants are shown in Fig. 4. The two key funnels are conserved, although a number of differences arise, for example stabilisation of the alternative Ub-CR configurations in the L67S mutant. The deviation between the canonical Ub and Ub-CR structures for all of the mutants is small (see

supporting information). Interestingly, the two sequences that give the best experimental differentiation between $\mathrm{Ub}$ and $\mathrm{Ub}-\mathrm{CR}$, with significant occupation equilibrium populations for both states without multiple phosphorylation events, namely F4A and T66V+L67N, are closest to the wild type landscape.

\section{Changes to the Ub to Ub-CR mechanism}

In all the mutants we observe conservation of the overall free energy barrier at high temperatures, although the relative energies of the two minima and the transition state change.

For the L67S mutant we observe significant stabilisation of the Ub-CR configuration, in agreement with experiment. ${ }^{13}$ The key change in the transition mechanism is the increased flexibility of Ser67 due to the smaller excluded volume, as well as the polarity of the side chain. Ser67 can move partly out of the hydrophobic pocket in the Ub configuration, a process favoured by solvent interaction and entropy. The motion of residue 67 then pulls the entire strand out of the fold, initiating the loss of interactions, and forcing the opening of the scaffold. While in the wild type we observe a sequential loss of interactions, for the mutant 

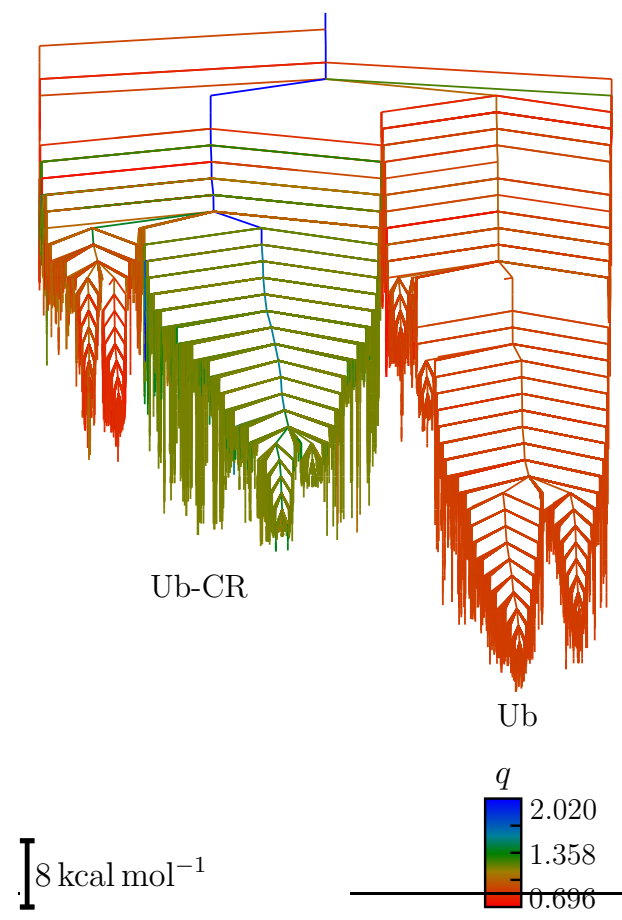

F4A mutant

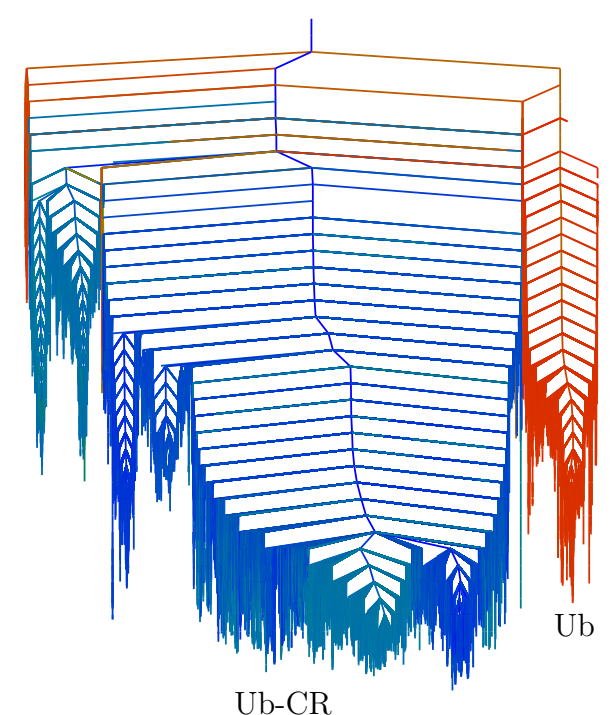

Ub-CR

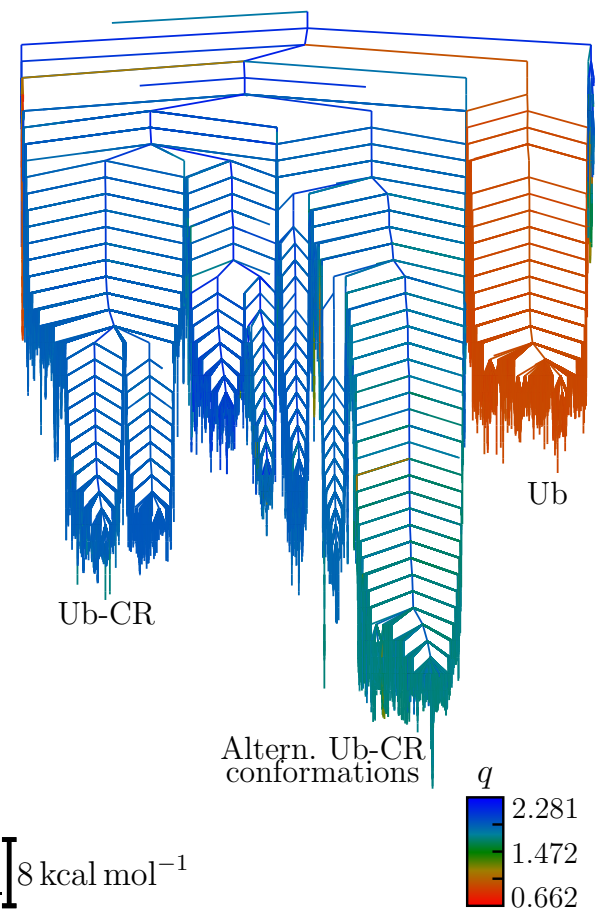

L67S mutant

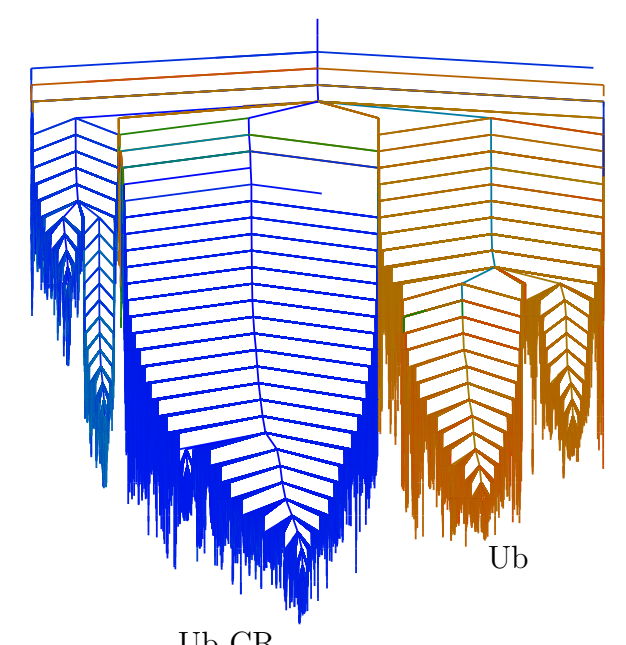

$\mathrm{Ub}-\mathrm{CR}$

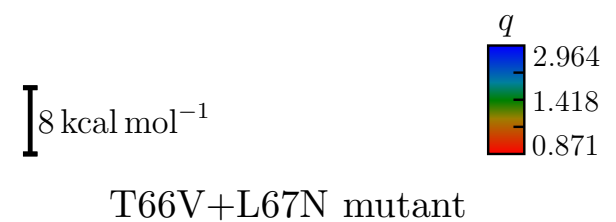

Figure 4: The disconnectivity graphs of the potential energy landscapes for the four mutants studied by Gladkova et al., coloured using the order parameter $q$. The two main funnels are conserved, however a number of alternative structures appear in the landscapes. 
the loss is more concerted, as the Ub configuration is destabilised. The interactions of Ser65 are conserved in this process, and only after the retraction is completed, can Ser65 and Ser67 change their relative positions leading to the canonical Ub-CR fold. This change has a number of consequences. It stabilises the alternative Ub-CR configurations, so they should be populated in this mutant. Furthermore, there are stable configurations with exposure of Ser65, Thr66 or Ser67, which would explain the observed multiple phosphorylation events for this sequence. In addition, at low temperatures (below $30^{\circ} \mathrm{C}$ ) an intermediate state is stabilised, further lowering the occupation probability of Ub.

For the F4A mutant only small changes are observed. Due to the smaller excluded volume around Ala4, and the lower propensity for $\beta$-sheet formation, the energetic penalty for the loss of interactions between residues 4 and 67 is lower. The retraction can therefore occur while Leu67 and Ala4 are still in contact, resulting in a lower barrier and a smaller number of backbone rearrangements after the retraction. The F4A mutation was introduced to shift the equilibrium towards UbCR, which is indeed observed in experiment, ${ }^{13}$ and consistent with the change in our predicted mechanism due to the lack of stabilisation of Ub by Phe4-Leu67 interactions observed in the wild type.

For the $\mathrm{T} 66 \mathrm{~V}+\mathrm{L} 67 \mathrm{~N}$ mutant a key change is observed in the Ub configuration. While Leu67 interacts through the backbone with Phe4, Asn67 can instead interact through the side chain. This switch destabilises the Ub conformation, as it pulls the strand partly out of the hydrophobic pockets. Additionally, the interactions between the two residues are conserved during the retraction, stabilising the transition state. This mutant has also been used by Schubert et. al ${ }^{15}$ to study the complex between PINK1 and Ub-CR, and we here confirm its validity as an enhanced PINK1 model substrate.

The case of the L71Y mutant is a curious one, as experimentally the Ub-CR configuration was not seen, yet the energy landscape picture confirms the likely population of this structure. However, the mutation leads to interactions between residue 71 and the $\beta 1 / \beta 2$-loop, even in the Ub-CR structure. The main effect of these additional interactions is a much larger 
energy barrier, probably making the transition very slow. In experiment this mutation was used to stabilise the Ub conformation, ${ }^{13}$ which agrees with the changed mechanism, lowering the exchange rate.

\section{Predicted exchange rates}

As for the wild type, an estimate of the free energy barriers may be calculated from experimental data, and compared to the computed barriers from the energy landscape. For the F4A mutant, the experimental barriers are $17.4 \mathrm{kcal} \mathrm{mol}^{-1}$ and $19.1 \mathrm{kcal} \mathrm{mol}^{-1}$ at $45^{\circ} \mathrm{C}$ and $25^{\circ} \mathrm{C}$, respectively, compared to our estimates of $17.7 \mathrm{kcal} \mathrm{mol}^{-1}$ and $18.9 \mathrm{kcal} \mathrm{mol}^{-1}$, which correspond to differences of less than $k_{\mathrm{B}} T$. For the T66V $+\mathrm{L} 67 \mathrm{~N}$ mutant, our computed barrier of $13.0 \mathrm{kcal} \mathrm{mol}^{-1}$ also compares well with the experimental barrier of $15.8 \mathrm{kcal} \mathrm{mol}^{-1}$. Our calculated exchange rate for the L71Y mutant is $10^{-4} \mathrm{~s}^{-1}$. Even if the free energy barrier was overestimated by $5 \mathrm{kcal} \mathrm{mol}^{-1}$, the rate would be two orders of magnitude smaller than for the native sequence. These predictions imply that the transition could not be resolved on the experimental time scale.

\section{Conclusions}

We have explored the potential and free energy surfaces for ubiquitin and four ubiquitin mutants, to determine the mechanism for the Ub to Ub-CR transition at an atomistic level of detail. The pathway is characterised by the loss of all interactions between the C-terminal tail and the surrounding residues prior to retraction, opening of the ubiquitin scaffold in a clam-like fashion, and a concerted process associated with a well-defined effective free energy barrier. Our results are in excellent agreement with reported experimental data. In addition, we provide detailed explanations for observed features of the $\mathrm{Ub} / \mathrm{Ub}-\mathrm{CR}$ system. We propose the existence of alternative Ub-CR conformations with Ser65 unexposed, and based on their stability in the L67S mutant, these structures should be observable. Furthermore, we suggest 
an explanation for the observed differences in phosphorylation behaviour between L67S and T66V +L67N, since the L67S mutant exhibits stable configurations with exposure of Ser65, Thr66 or Ser67, while in T66V + L67N the interactions between Asn67 and Phe4 protect the residues, aside from Ser65. Finally, we have calculated the exchange rate for L71Y, which explains why Ub-CR has not been detected for this mutant.

\section{Acknowledgement}

The authors thank the EPSRC for financial support.

\section{Supporting Information Available}

The research data is available at doi:10.5281/zenodo.1254354. The supporting information contains: Deviations from crystal structures; solvent exposure of key residues for wild type ubiquitin; details of the Ub to Ub-CR transition in wild type ubiquitin; Arrhenius behaviour for the wild type. This material is available free of charge via the Internet at http: $/ /$ pubs.acs.org/.

\section{References}

(1) Komander, D., and Rape, M. (2012) The ubiquitin code. Annu. Rev. Biochem. 81, 203-229.

(2) Ordureau, A., Münch, C., and Harper, J. (2015) Quantifying ubiquitin signaling. Mol. Cell 58, 660-676.

(3) Swatek, K. N., and Komander, D. (2016) Ubiquitin modifications. Cell Res. 26, 399422.

(4) Yau, R., and Rape, M. (2016) The increasing complexity of the ubiquitin code. Nat. Cell Biol. 18, 579-586. 
(5) Pickrell, A., and Youle, R. (2015) The roles of PINK1, Parkin, and mitochondrial fidelity in Parkinson's disease. Neuron 85, 257-273.

(6) Nguyen, T. N., Padman, B. S., and Lazarou, M. (2016) Deciphering the molecular signals of PINK1/Parkin mitophagy. Trends Cell Biol. 26, 733-744.

(7) Pickles, S., Vigié, P., and Youle, R. J. (2018) Mitophagy and Quality Control Mechanisms inÂăMitochondrial Maintenance. Curr. Biol. 28, R170-R185.

(8) Harper, J. W., Ordureau, A., and Heo, J.-M. (2018) Building and decoding ubiquitin chains for mitophagy. Nat. Rev. Mol. Cell Biol. 19, 93-108.

(9) Kazlauskaite, A., Kondapalli, C., Gourlay, R., Campbell, D., Ritorto, M., Hofmann, K., Alessi, D., Knebel, A., Trost, M., and Muqit, M. K. (2014) Parkin is activated by PINK1-dependent phosphorylation of ubiquitin at Ser65. Biochem. J. 460, 127-141.

(10) Kane, L. A., Lazarou, M., Fogel, A. I., Li, Y., Yamano, K., Sarraf, S. A., Banerjee, S., and Youle, R. J. (2014) PINK1 phosphorylates ubiquitin to activate Parkin E3 ubiquitin ligase activity. J. Cell Biol. 205, 143-153.

(11) Koyano, F., Okatsu, K., Kosako, H., Tamura, Y., Go, E., Kimura, M., Kimura, Y., Tsuchiya, H., Yoshihara, H., Hirokawa, T., Endo, T., Fon, E. A., Trempe, J.-F., Saeki, Y., Tanaka, K., and Matsuda, N. (2014) Ubiquitin is phosphorylated by PINK1 to activate parkin. Nature 510, 162-166.

(12) Wauer, T., Swatek, K. N., Wagstaff, J. L., Gladkova, C., Pruneda, J. N., Michel, M. A., Gersch, M., Johnson, C. M., Freund, S. M., and Komander, D. (2015) Ubiquitin Ser65 phosphorylation affects ubiquitin structure, chain assembly and hydrolysis. EMBO J. 34, 307-325.

(13) Gladkova, C., Schubert, A. F., Wagstaff, J. L., Pruneda, J. N., Freund, S. M. V., 
and Komander, D. (2017) An invisible ubiquitin conformation is required for efficient phosphorylation by PINK1. EMBO J. e201797876.

(14) Dong, X., Gong, Z., Lu, Y.-B., Liu, K., Qin, L.-Y., Ran, M.-L., Zhang, C.-L., Liu, Z., Zhang, W.-P., and Tang, C. (2017) Ubiquitin S65 phosphorylation engenders a pHsensitive conformational switch. Proc. Natl. Acad. Sci. USA 114, 6770-6775.

(15) Schubert, A. F., Gladkova, C., Pardon, E., Wagstaff, J. L., Freund, S. M. V., Steyaert, J., Maslen, S. L., and Komander, D. (2017) Structure of PINK1 in complex with its substrate ubiquitin. Nature 552, 51-56.

(16) Valente, E. M., Abou-Sleiman, P. M., Caputo, V., Muqit, M. M. K., Harvey, K., Gispert, S., Ali, Z., Del Turco, D., Bentivoglio, A. R., Healy, D. G., Albanese, A., Nussbaum, R., González-Maldonado, R., Deller, T., Salvi, S., Cortelli, P., Gilks, W. P., Latchman, D. S., Harvey, R. J., Dallapiccola, B., Auburger, G., and Wood, N. W. (2004) Hereditary Early-Onset Parkinson's Disease Caused by Mutations in PINK1. Science 304, 1158-1160.

(17) Wales, D. J. (2002) Discrete path sampling. Mol. Phys. 100, 3285-3305.

(18) Wales, D. J. (2004) Some further applications of discrete path sampling to cluster isomerization. Mol. Phys. 102, 891-908.

(19) Noé, F., and Fischer, S. (2008) Transition networks for modeling the kinetics of conformational change in macromolecules. Curr. Opin. Struc. Biol. 18, 154-162.

(20) Wales, D. J. (2010) Energy landscapes: some new horizons. Curr. Opin. Struc. Biol. 20, 3-10.

(21) Trygubenko, S. A., and Wales, D. J. (2004) A doubly-nudged elastic band method for finding transition states. J. Chem. Phys. 120, 2082. 
(22) Henkelman, G., and Jónsson, H. (1999) A dimer method for finding saddle points on high dimensional potential surfaces using only first derivatives. J. Chem. Phys. 111, 7010.

(23) Henkelman, G., Uberuaga, B. P., and Jónsson, H. (2000) A climbing image nudged elastic band method for finding saddle points and minimum energy paths. J. Chem. Phys. 113, 9901.

(24) Munro, L. J., and Wales, D. J. (1999) Defect migration in crystalline silicon. Phys. Rev. B 59, 3969-3980.

(25) Strodel, B., and Wales, D. J. (2008) Free energy surfaces from an extended harmonic superposition approach and kinetics for alanine dipeptide. Chem. Phys. Lett. 466, 105115 .

(26) Carr, J. M., and Wales, D. J. (2008) Folding pathways and rates for the three-stranded $\beta$-sheet peptide Beta3s using discrete path sampling. J. Phys. Chem. B 112, 8760-8769.

(27) Becker, O. M., and Karplus, M. (1998) The topology of multidimensional potential energy surfaces: Theory and application to peptide structure and kinetics. J. Chem. Phys. 106, 1495-1517.

(28) Wales, D. J., Miller, M. A., and Walsh, T. R. (1998) Archetypal energy landscapes. Nature 394, 758-760.

(29) Joseph, J. A., Röder, K., Chakraborty, D., Mantell, R. G., and Wales, D. J. (2017) Exploring biomolecular energy landscapes. Chem. Commun. 53, 6974-6988.

(30) Maier, J. A., Martinez, C., Kasavajhala, K., Wickstrom, L., Hauser, K. E., and Simmerling, C. (2015) ff14SB: Improving the accuracy of protein side chain and backbone parameters from ff99SB. J. Chem. Theory Comput. 11, 3696-3713. 
(31) Onufriev, A., Bashford, D., and Case, D. A. (2000) Modification of the generalized Born model suitable for macromolecules. J. Phys. Chem. B 104, 3712-3720.

(32) Onufriev, A., Bashford, D., and Case, D. A. (2004) Exploring protein native states and large-scale conformational changes with a modified generalized born model. Proteins 55, 383-394.

(33) Srinivasan, J., Trevathan, M. W., Beroza, P., and Case, D. A. (1999) Application of a pairwise generalized Born model to proteins and nucleic acids: inclusion of salt effects. Theor. Chem. Acc. 101, 426-434.

(34) Götz, A. W., Williamson, M. J., Xu, D., Poole, D., Le Grand, S., and Walker, R. C. (2012) Routine microsecond molecular dynamics simulations with AMBER on GPUs. 1. Generalized Born. J. Chem. Theory Comput. 8, 1542-1555.

(35) Salomon-Ferrer, R., Götz, A. W., Poole, D., Le Grand, S., and Walker, R. C. (2013) Routine microsecond molecular dynamics simulations with AMBER on GPUs. 2. Explicit Solvent Particle Mesh Ewald. J. Chem. Theory Comput. 9, 3878-3888.

(36) Mantell, R. G., Pitt, C. E., and Wales, D. J. (2016) GPU-accelerated exploration of biomolecular energy landscapes. J. Chem. Theory Comput. 12, 6182-6191.

(37) Vijay-Kumar, S., Bugg, C. E., and Cook, W. J. (1987) Structure of ubiquitin refined at 1.8 A resolution. J. Mol. Biol. 194, 531-44.

(38) Vallurupalli, P., Bouvignies, G., and Kay, L. E. (2012) Studying "invisible" excited protein states in slow exhange with a major state conformation. J. Am. Soc. Chem. $134,8148-8161$.

(39) Hospenthal, M. K., Freund, S. M. V., and Komander, D. (2013) Assembly, analysis and architecture of atypical ubiquitin chains. Nat. Struct. Mol. Biol. 20, 555-565. 


\section{Graphical TOC Entry}

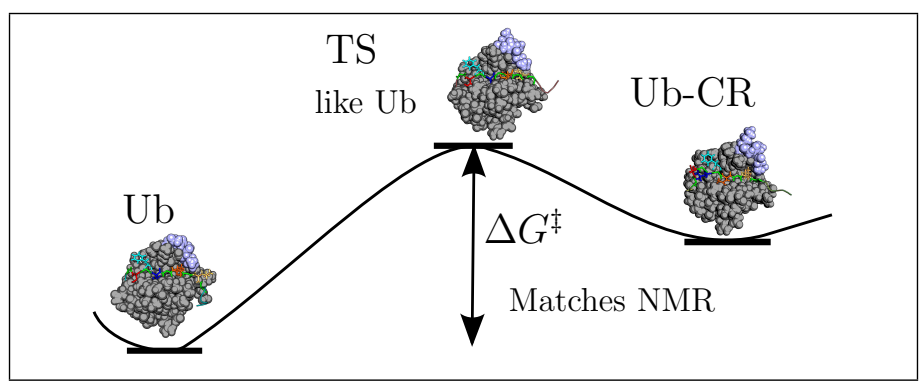

